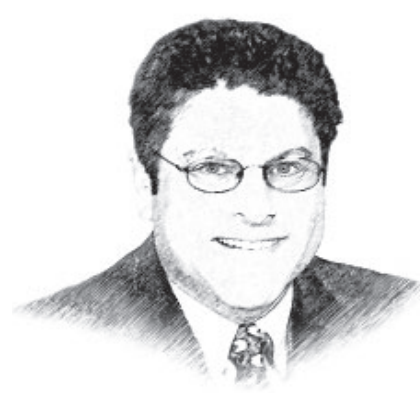

\title{
Perioperative infection: Are we sure what to focus on?
}

The relationship between immunosuppressants and infection is complex. True, these medications can increase the risk of opportunistic and perhaps routine infections. Some of the newer drugs increase the risk of reactivation of tuberculosis (and others are assumed to do so), some appear to increase the risk of activation of $\mathrm{JC}$ virus (an almost ubiquitous asymptomatic infection), and some predispose to Pneumocystis pneumonia or herpes zoster. But different agents do not equally increase the risk. Steroid therapy is the least precise weapon in our immunosuppressive arsenal and likely provokes the widest array of infections. We all are aware of this, but we continue to be gravely concerned about the risk of the newer biologics.

Then there is the seeming paradox that we can treat some severe infections with adjunctive immunosuppression; examples include bacterial meningitis, tuberculous pericarditis, Pneumocystis infection, and septic arthritis. Studies are under way using anti-interleukin 6 drugs to treat COVID-19 systemic inflammatory syndrome, so-called cytokine storm. We cannot assume that immunosuppression is always deleterious in the setting of otherwise appropriately managed infection, and specific scenarios need to be evaluated with attention to all potential confounders.

I don't imply that our newer biologics pose no significant risk of infection. They clearly do, although for some, I feel that the greater risk is that they mask clinical and laboratory signs of early infection. Thus, patients are actually sicker by the time the infection is recognized. For others, the body's ability to resolve a specific infection can indeed be significantly decreased. The different targeted inflammatory molecules and cells perform different roles in the inflammatory opera. We should not assume that disabling one will have the same effect as disabling another.

The discussion of whether to continue or withhold (and if so, for how long) immunosuppressive drugs before elective surgery has been going on for years. Two highinterest scenarios have been elective arthroplasty in patients with rheumatoid arthritis (RA) and abdominal surgery in patients with inflammatory bowel disease (IBD). Moosvi et al, in this issue of the Journal (page 343), weigh in with a discussion of whether to withhold biologics in patients undergoing intra-abdominal surgery for IBD. They argue nay for the most frequently used biologics, based on mixed and insufficient evidence of benefit of withholding, including a recent large nonrandomized prospective study of anti-tumor necrosis factor (TNF) agents. Interestingly, this trial ${ }^{1}$ included analysis of whether the anti-TNF agents were detectable in the blood, which made no difference in risk of infection.

This recommendation differs from recent guidelines for management of biologics in the setting of elective arthroplast $y^{2}$ that suggested holding these drugs for one dosing cycle in advance of planned surgery. The evidence-based medicine jury has not weighed in that there definitely is an increased risk of infection if the drug is continued, as no large randomized prospective trial has compared continuing vs withholding the drug before arthroplasty, and it is not fair to compare outcomes of patients who 
have been on biologic therapy with those who have never been on comparable therapy. Delaying one dose before totally elective joint surgery is often not associated with a major flare in inflammatory arthritis, but that may not be the case with an IBD patient requiring more semielective surgery. Concern over provoking a flare in the underlying disease, which may require steroid therapy to manage, has been an argument against withholding medications before elective surgery.

The historically conservative recommendations to withhold biologics before surgery are based on the fear of postoperative infection, especially of a prosthetic joint, in the absence of ideal data demonstrating safety.

But this reasoning has perhaps paid insufficient attention to the effect of corticosteroids on surgical and clinical outcomes. Steroid therapy has always been a known confounder of outcome studies, particularly of surgical outcome. Although it is well established that steroids are associated with increased risk of suboptimal outcomes of arthroplasty, patients with IBD, like those with RA, who need surgery to manage a complication of their disease have experienced, on average, more severe disease and have likely needed corticosteroid therapy. Recently, George et $\mathrm{al}^{3}$ performed a retrospective review using Medicare administrative data of RA patients taking biologics and undergoing elective hip or knee surgery. There was no difference between the biologics. The authors did not assess whether use of any biologic increased the risk compared with patients who had never been on a biologic or if the biologic had been withheld preoperatively, although at least for intravenous infliximab that doesn't seem to make a big difference, ${ }^{4}$ so this study doesn't shed direct light on the question addressed by Moosvi et al in this issue. However, the striking observation of George et $\mathrm{al}^{3}$ was affirmation that even low doses of corticosteroids (prednisone equivalent $>5 \mathrm{mg}$ ) increased the risk of various postoperative infections and readmission within 30 days.

I don't know if lowering the steroid dose preoperatively to less than $5 \mathrm{mg}$ will decrease that risk of infection, nor do I know whether patients will be at higher risk if they experience a flare in their IBD or arthritis and require a slight bump in their steroid dose due to withholding their biologic preoperatively. But I believe that we may have been a bit off target as we have focused so much on the biologics, and less on what we assumed to be low and safe doses of perioperative steroids.



BRIAN F. MANDELL, MD, PhD Editor in Chief

1. Cohen BL, Fleshner P, Kane SV, et al. 415a-Anti-tumor necrosis factor therapy is not associated with post-operative infection: results from prospective cohort of ulcerative colitis and Crohn's disease patients undergoing surgery to identify risk factors for postoperative infection I (PUCCINI) [abstract]. Gastroenterology 2019; 156(6 suppl 1):S80. doi:10.1016/S0016-5085(19)36987-2

2. Goodman SM, Springer B, Guyatt G, et al. 2017 American College of Rheumatology/American Association of Hip and Knee Surgeons Guideline for the perioperative management of antirheumatic medication in patients with rheumatic diseases undergoing elective total hip or total knee arthroplasty. J Arthroplasty 2017; 32(9):2628-2638. doi:10.1016/j.arth.2017.05.001

3. George MD, Baker JF, Winthrop K, et al. Risk of biologics and glucocorticoids in patients with rheumatoid arthritis undergoing arthroplasty. A cohort study. Ann Intern Med 2019; 170(12):825-836. doi:10.7326/M18-2217

4. George MD, Baker JF, Hsu JY, et al. Perioperative timing of infliximab and the risk of severe infection after elective hip and knee arthroplasty. Arthritis Care Res (Hoboken) 2017; 69(12):1845-1854. doi:10.1002/acr.23209 